\title{
Effect of the Colombian Emergency Department Triage Policy on the timeliness of ED care and the mortality in patients with cerebrovascular attack: $A$ controlled interrupted time series analysis
}

Carlos Eduardo Vallejo ( $\square$ eduardo.vallejo@udea.edu.co)

Universidad de Antioquia https://orcid.org/0000-0001-5677-8557

Daniel Felipe Patiño-Lugo

Universidad de Antioquia

Daniel Camilo Aguirre-Acevedo

Universidad de Antioquia

Juan Pablo Acosta

Hospital Pablo Tobon Uribe

Research article

Keywords: Ischemic Cerebrovascular Attack, Triage, Quality of Health Care, Emergency Treatment, Mortality, Interrupted Time Series Analysis

Posted Date: August 17th, 2020

DOI: https://doi.org/10.21203/rs.3.rs-55373/v1

License: (1) This work is licensed under a Creative Commons Attribution 4.0 International License.

Read Full License 


\section{Abstract}

Background: Ischemic Cerebrovascular Accident (CVA) is the second cause of death and one of the leading causes of disability in the world. In Colombia, there is a prevalence of $0.16 \%$ and a mortality rate of 16.82 deaths per 100,000 inhabitants. This study assessed the Colombian Ministry of Health's Emergency Department Triage Policy (TP) on the timeliness of hospital care, the mortality, and change in reperfusion therapy of patients with the first episode of an CVA in the Emergency Department (ED) of a hospital in the city of Medellín.

Methods: A controlled interrupted time series analysis between January 2011 and November 2017 was performed in one emergency department using segmented regression analysis. The emergency department of other hospital was used as a control. Data were aggregated by month for both ED, including 60 pre-intervention and 23 intervention points.

Results: No decrease in the timeliness of care in the ED was found in patients with a stroke after implementing the Colombian Ministry of Health's TP (12.55 minutes, IC -17.07, 42.17; p: 0.4). The TP intervention produced a level change in the timeliness of care in Triage I and II subgroups, of 28.34 minutes (IC 95\% 16.9, 39.79; p valor 0.00), there was no change in the trend. The classification of Triage I and II was more frequent in the post-intervention period. There was evidence of the increase in reperfusion therapy with tissue plasminogen activator (rTPA) in $4 \%(95 \% \mathrm{Cl}-0.08,-0.01$; $p$ value 0.01$)$. Mortality increased $4 \%$ in the post-intervention period without being statistically significant $(0.04$, IC $95 \%$ $0.08-0 ; p$ valor 0.06 ). There were no significant changes in the same outcomes in the control group.

Conclusions: The implementation of the triage policy allowed improving the timeliness of ED care only in patients with ischemic CVA classified as Triage I and II, in an Emergency Department in the city of Medellín, Colombia.

\section{Key Message:}

\section{What Is Already Known on this Topic:}

- CVA is a time-sensitive disease where the timeliness of ED care is definitive to reduce the high morbidity and mortality; therefore, It is a disease that must be detected and managed at Emergency Departments. In Colombia, the Ministry of Health decreed a structured five-level triage policy with the purpose of improving the timeliness of ED care and reducing the morbidity and mortality cerebrovascular attack.

- At present, there are no data to establish the effect of this triage policy on "time-sensitive" diseases, such as cerebrovascular attack, in which we hope to obtain the greatest benefit. Therefore, this study evaluates the effect of the triage policy on the timeliness of ED care, the incidence of in-hospital mortality, and the change in the frequency of reperfusion therapy of patients with the first episode of acute CVA in the Emergency Department. 


\section{What This Study Adds:}

- This study shows the improvement in the timeliness of ED care of the population classified as triage I and II, with ischemic stroke that consulted the ED of the hospital where the study was conducted.

- There is evidence of change in the proportion of patients classified in Triage Categories for more timely care (I and II, Triage Categories), and there is evidence of an increase in the frequency of reperfusion therapy in this population. But this study showed no change in mortality.

\section{Background:}

The ischemic Cerebrovascular Accident (CVA) is the second cause of death and one of the leading causes of disability in the world [1]. For the period of 2002 to 2008, the incidence of CVA in low and middle income countries was $20 \%$ more than in high-income countries [2], [3]. In Colombia, in 2014, an estimated prevalence of $0.16 \%, 3,702$ deaths related to stroke, and a mortality rate of 16.82 deaths per 100,000 inhabitants, were reported [4].

CVA is a "time-sensitive" disease, so alterations in the timeliness of ED care increases the risk of adverse outcomes [5]. Structured triage systems in the Emergency Department (ED) are a critical intervention to prioritize patient care in order to improve the timeliness of ED care [6], [7]. With the intention of improve the timeliness of ED care, in December 2015, the Colombian Ministry of Health decreed a Triage Policy [8]. This policy is strictly enforced and aims to improve the timeliness of ED care and reduce the risk of death [8].

This study aims to evaluate the effect of the TP on the timeliness of ED care, the incidence of in-hospital mortality, and the frequency of use of reperfusion therapy in patients with the first episode of acute ischemic CVA in the ED of a hospital in the city of Medellín.

\section{Methods:}

\section{Study Design and Justification}

A controlled interrupted time series (ITS) analysis with a segmented regression analysis was performed [9]-[14]. This design evaluates the effect of an intervention when there is no possibility of randomization [15]. It has been used in the evaluation of complex interventions with clearly identified pre- and postintervention periods [10], [16]-[19]. Control was established with a hospital that did not implement the policy during the study period because it had a similar triage system since 2011; the use of the control allows controlling the effect of possible time-related not observed confuners, which are not controlled with ITSs without control groups [14].

\section{Participants}


Cases were defined as patients with the first episode of an ischemic CVA between January 2011 and November 2017, in which the diagnosis of admission to the ED and hospital discharge was CVA or its equivalent (see the International Classification of Diseases (ICD)-10 Codes we used, Appendix 1).

The medical records were reviewed for data verification, eligibility and extraction of the following information: identification, age, sex, date of emergency consultation, time of triage, door to doctor time, assigned triage category, type of health insurance, performing intravenous thrombolysis with tissue plasminogen activator (rTPA) and vital status at discharge (alive or dead). Cases with diagnoses other than CVA were excluded.

This study was conducted in a high-complexity hospital in the city of Medellín, which is not specialized in the management of CVA. It has an ED with 120 gurneys, general medical staff and specialists in emergency medicine, and on-call medical specialties and sub-specialties. CT Scans are available 24 hours a day outside the ED. It has hospitalization service with more than 600 beds and an ICU. It has a permanent triage service managed by nurses. The control group was another hospital in the city with similar technical and human characteristics for the care of patients with CVA. This hospital implemented the structured five-level triage in years prior (2013) to the entry into force of the Ministry of Health policy.

The data requested was handled by the researchers and was stored in an Excel file for analysis.

\section{Intervention}

The intervention was the implementation of the triage policy developed by the Ministry of Health, which generated a change in the way of performing triage in the studied hospital. The characteristics of the triage before and after the disclosure of the triage policy are shown in Fig. 1S - Appendix 2.

The post-intervention period began in January 2016, when the triage policy became a mandatory compliance policy [8]. This policy dictates technical provisions about triage. In the Triage I and II classification, care times are assigned, which must be immediate and less than 30 minutes, respectively; for Triage III, IV and V, information on the average care time should be given without a target time. In the institution where this study was conducted, formal training of the personnel in charge of triage was carried out during the months of November and December 2015, based on the recommendations of the American College of Emergency Physicians (ACEP).

\section{Outcomes}

The main outcome was timeliness of ED care, defined as the mean time in minutes from the completion of the triage to medical care (Eq. 1 - Appendix 1). The secondary outcome was the in-hospital mortality of patients admitted with a diagnosis of CVA, expressed as a proportion in each measurement point (Eq. 2 - Appendix 1). Additionally, the change in the use of rTPA in this population in the periods studied was estimated, and the outcomes were treated as aggregated data.

\section{Study Period, Sample and Statistical Power}


The study period comprised January 2011 to November 2017. The measurements were taken in aggregate (averages or proportions) in each month during this period of time. These aggregated data at each measurement point constitute our unit of analysis [12].

For the study period, measurement points were taken during two-time segments: the first segment called pre-intervention - occurred from January 2011 to December 2015; the second segment - called post-intervention - occurred from January 2016 to November 2017. The sample size corresponds to 83 months of measurement, distributed as follows: 60 months prior to the implementation of the strategy and 23 months from the implementation of the strategy.

The statistical power of our study was calculated using the scenarios proposed by Zhang et al. [20], which takes into account the number of measurement points before and after the intervention, the autocorrelation, and the size of the expected effect. For example, with a sample of at least 72 measurement points, where $2 / 3$ are pre-intervention, a power greater than $80 \%$ can be obtained if the autocorrelation is less than 0.4 and the effect size is 0.5 [20]. The size of the effect on the ITSs refers to the level change in the outcome after the intervention, to the change in trend or both [21].

\section{Data Analysis}

Descriptive analysis of demographic, clinical and organization of care characteristics was performed. For the ITS analysis we used the robust ITS model developed by Cruz et al. [22], which distinguishes between the moment in which the intervention is performed and the moment in which a change in the outcome occurs and allows to detect the pre- and post-intervention differences of autocorrelation and variances. Traditional models assume that autocorrelation and variance are constant in both periods, which does not represent the cases in which the intervention aims to reduce the variability in the system. In our case, the intervention aims to improve the health care process; therefore, the effects are expected to be more dependent and consistent over time; therefore, autocorrelation and variance must be different after the intervention[22].

This model uses ordinary least-square (OLS) regressions to estimate the following parameters of interest: the intercept at the beginning of the time period, the slope of the trend before the intervention, the intercept of the post-intervention phase and the slope of the post-intervention trend. With this information, the level change and the change in the slope are calculated [22].

Subgroup analysis was performed according to the triage classification. The change in the use of rTPA in the periods of time studied was estimated. Other analyses were also carried out by age, gender, type of health insurance and shift of care for the outcomes in care and mortality (see Appendix 2).

All the analyses were performed with the Robust ITS Software [23] and with the R Software, version 1.1.383 and the packages for approximation by autoregressive methods, if necessary. The packages used were: tidyr, readxl, car, questionr, MASS, forecast, auto.arima, stats, Im, glm, graphics. 
This study was approved by the Technical and Ethical Committees of the hospitals where the study was conducted.

\section{Results:}

A total of 5,763 cases were found, 3,101 exclusions were made, 1,051 cases for previous hospital admission for the same cause and 2,040 cases for diagnoses other than CVA. A total of 2,662 cases met criteria for admission to the study and analysis (See flowchart in Diagram 1). There were 2,009 cases during the pre-intervention period and 653 , during the post-intervention period.

Table 1 presents the demographic, clinical and organization of care characteristics. It shows a homogeneous distribution in the groups of both periods in the variables age, sex, insurance and day or night care. In both periods, the average age was 75 years old. The proportion of women was $52 \%$ in the pre-intervention period and $55 \%$ in the post-intervention period. The proportion of individuals in the tax system was $75 \%$ in both periods. Care was more frequent during the day than at night in the whole time series, but an increase is evidenced in the care at night, with a frequency of $30 \%$ in the pre-intervention period vs. $40 \%$ in the post-intervention period. There was a change in the distribution of the classification of triage groups: Categories I (22.51\%) and II (48.045\%), in the post-intervention period, compared to the pre-intervention period ( $5.27 \%$ and $28.87 \%$, respectively). There was also an increase in the frequencies of reperfusion therapy with rTPA (0.6\%, pre-intervention vs. $11.9 \%$ post-intervention). See Table 1. 


\begin{tabular}{|c|c|c|}
\hline & Pre-Int & Post-Int \\
\hline & $\mathrm{n}=2,009$ & $\mathrm{n}=653$ \\
\hline Female Sex n (\%) & $1,036(52 \%)$ & $353(55 \%)$ \\
\hline Age (median / IQ25 - IQ75) & $75.0(64-83)$ & $76.0(67-84.0)$ \\
\hline Vital status: dead n (\%) & $198(9.8 \%)$ & $84(12 \%)$ \\
\hline Thrombolysis: Yes n (\%) & $14(0.6 \%)$ & $78(11.9 \%)$ \\
\hline Contributive Insurance Company n (\%) & $1,499(74.61 \%)$ & $485(74.27 \%)$ \\
\hline Subsidized Insurance Company n (\%) & $510(25.38 \%)$ & $168(25.72 \%)$ \\
\hline Day Shift n (\%) & $1,394(69.38 \%)$ & $382(58.95 \%)$ \\
\hline Night Shift n (\%) & $615(30.61 \%)$ & $271(41.50 \%)$ \\
\hline Triage I n (\%) & $106(5.27 \%)$ & $147(22.51 \%)$ \\
\hline Triage II n (\%) & $580(28.87 \%)$ & $314(48.08 \%)$ \\
\hline Triage III n (\%) & $1,161(57.78 \%)$ & $185(28.33 \%)$ \\
\hline Triage IV n (\%) & $106(5.27 \%)$ & $5(0.76 \%)$ \\
\hline Triage V n (\%) & $56(2.78 \%)$ & $2(0.3 \%)$ \\
\hline
\end{tabular}

Pre-Int $=$ Pre-Intervention Period $;$ Post-Int $=$ Post-Intervention Period $;$ IQ = Interquartile Range, $n=$ Group Sample; $\%=$ percentage

\section{Timeliness of care in ED}

In Fig. 1, the timeliness of ED care is plotted in average time each month. The results indicate that, at the beginning of the observation period, the timeliness of ED care was 50.37 minutes (Standard Deviation (SD) 11.3). Before the intervention, the slope had a negative slope $(-0.1$ minutes, $p$ value $=0.43)$. Immediately after the intervention, a level change was identified, since the timeliness of ED care was 44.61 minutes at the end of the pre-intervention period and 32.06 minutes at the beginning of the postintervention period (a level difference of 12.55 minutes, $95 \% \mathrm{Cl}-17.07,42.17$; p: 0.4 ). The slope in the postintervention period is positive, at 0.01 minutes. The change in the slope between the pre- and postintervention period is 0.11 minutes $(95 \% \mathrm{Cl}-1.9,2.12 ; \mathrm{p}$ : 0.91). See Table 2 with a summary of parameters and Appendix 2 for details of the model. In the control group, there were no significant changes in the level or in the trend in timeliness of ED care (Fig. 2). 
Table 2

95\% Confidence Intervals and Average Parameter Estimates

\begin{tabular}{|c|c|c|c|c|}
\hline & \multicolumn{2}{|c|}{ Intervention Group } & \multicolumn{2}{|c|}{ Control Group } \\
\hline & Opportunity & $\begin{array}{l}\text { T I \& II } \\
\text { Opportunity }\end{array}$ & Opportunity & $\begin{array}{l}\text { T I \& II } \\
\text { Opportunity }\end{array}$ \\
\hline $\begin{array}{l}\text { Intercept of the pre-intervention } \\
\text { point }\end{array}$ & 50.37 & 31.05 & 19.42 & 3.48 \\
\hline $\begin{array}{l}\text { Intercept of the post-intervention } \\
\text { point }\end{array}$ & 31.32 & 25.84 & 37,85 & 4.14 \\
\hline Change in intercepts, $\delta$ & 19.05 & -0.39 & -18.43 & -0.66 \\
\hline Change in level, $-\delta \nabla-\Delta \nabla \tau \square$ & $\begin{array}{l}12.55(-17.07 \\
42.12)\end{array}$ & $\begin{array}{l}28.34 \text { (16.9, } \\
39.79)\end{array}$ & 17,88 & 2.2 \\
\hline Slope pre-intervention point & -0.1 & 0.29 & -0.04 & 0 \\
\hline Slope post-intervention point & 0.01 & -0.1 & -0.44 & 0 \\
\hline Change in the slope, $\Delta \square$ & $\begin{array}{l}0.11(-1.9 \\
2.12)\end{array}$ & $\begin{array}{l}-0.39(-0.91 \\
0.13)\end{array}$ & $\begin{array}{l}-0.4(-0.82 \\
0.03)\end{array}$ & $\begin{array}{l}-0-08(-0.29, \\
0.03)\end{array}$ \\
\hline $\begin{array}{l}\text { Delay in the effect of the } \\
\text { intervention, - } \tau \mathbb{-} t\end{array}$ & -2 & -2 & 0 & 0 \\
\hline
\end{tabular}

\section{In-hospital mortality}

In the outcome of in-hospital mortality in patients with ischemic CVA, before the start of the observation period (zero time), the mortality was $8 \%$. Before and after the intervention, there was no change in the slopes; both were zero (0). Immediately after the intervention, a $3 \%$ change in the level in mortality was identified $(95 \% \mathrm{Cl}-0.08,0.01 ; \mathrm{p}: 0.12)$. The autocorrelation coefficient of this model was 0.1 preintervention and -0.51 , post-intervention, so there was no approximation with autoregressive models. See the model detail in Fig. $3 S$ - Appendix 2.

\section{Triage classification and subgroup analysis:}

There was a change in the classification in triage groups in the ED in the population with ischemic CVA. In the post-intervention period, the Triage I and II groups increased, compared to the pre-intervention period (see Table 1 and Fig. 4S - Appendix 2). In the analysis by subgroups - the timeliness of ED care in the Triage I - II subgroup - it was shown that at the beginning of the observation period (zero time), the result was 31.05 minutes. Before and after the intervention, a difference of -0.39 was found in the trend, a slightly negative slope $(95 \% \mathrm{Cl}-0.91,0.13 ; \mathrm{p}$ valor 0.14$)$. Immediately after the intervention, a level change of 28.34 minutes was evidenced, passing from 48.11 minutes at the end of the pre-intervention period to 19.76 minutes at the beginning of the post-intervention period ( $95 \% \mathrm{Cl} 16.9,39.9 ;$ p: 0.00$)$. See Fig. 3 and Table 2 with the summary of parameters. The rest of the subgroup analysis for the timeliness of ED care 
and in-hospital mortality are presented in Appendix 2. In the control group, there were no significant changes in the change of trend or level in this outcome.

\section{Reperfusion therapy}

There was a change in the proportion of the use of rTPA for reperfusion treatment in the pre- and postintervention periods. Immediately after the intervention, a level change of -0.04 was identified, which means an increase of $4 \%$, rising from 0.02 at the end of the pre-intervention period to 0.06 at the beginning of the post-intervention period $(95 \% \mathrm{Cl}-0.08,-0.01$; $p$ value 0.01$)$. There were no significant changes in the trend. Figure 5S Appendix 2. The control group showed no changes in any of the outcomes studied (Table 2).

The autocorrelograms for each model are found around zero (see Appendix 2).

Confusion and interaction analyses were carried out, adjusting the model for the insurance, shift, age and sex variables, without finding variation in the calculated raw values (Appendix 2).

\section{Discussion:}

\section{Principal Findings}

The objective of this study was to evaluate the effect of the ED TP proposed by the Colombian Ministry of Health. The results show an improvement in the opportunity of care of the population with ischemic CVA that consulted the ED of the hospital where the study was conducted only in the population classified as Triage I and II. As for the mortality outcome, a post-intervention slope with a negative tendency was observed, without being statistically significant. Other relevant findings were the increase in the proportion of patients classified as Triage I and II and the increase in the use of rTPA for reperfusion therapy in the post-intervention period.

\section{Comparison of the Principal Findings with Other Studies The timeliness of ED care:}

The structured five-level triage has proven to be a useful tool to organize care and determine the timeliness for medical evaluation in the ED [24]-[26], [27], [28]. Timeliness is an indicator of the quality of care in the ED [5], since a poor timeliness in care is associated with adverse outcomes [5], [27]-[29] and "time-sensitive" diseases - such as CVA - present more frequent adverse outcomes when there is poor timeliness of ED care[30].

CVA requires timely attention and management. Saver et al. found that when an ischemic CVA occurs due to the obstruction of large vessels, an average of 1.9 million neurons, 14 billion synapses and 12 kilometers of myelin fibers are lost for every minute that passes since the beginning of the vascular obstruction [31]. Further, Meretoja et al. show that - for every minute saved when performing reperfusion 
therapy with rTPA - 1.8 extra days of healthy life are provided [32]. The American Heart Association and the American Stroke Association, recommend the implementation of triage protocol as a good practice to improve timelines of care in ED for patients with CVA [7]. Xian et al. evidenced that the implementation of a triage protocol in the ED decreased the acute door-to-needle time (DTN) by an average of 8.1 minutes [33]. Another study conducted by Xian et al. evidenced that the implementation of triage in hospitals defined as reference centers for the management of CVA decreases - 2.6 ( $\mathrm{Cl}-4-0$ a -1.3) times the time to perform reperfusion therapy compared to those centers that had not implemented a triage protocol [34]. Likewise, Lindsberg et al. demonstrated that the updating of triage improves the DTN by approximately 38 minutes, and evidenced an increase in the reperfusion therapy [35].

\section{Mortality}

Identifying the probable case is part of the chain of interventions in the care of CVA [7], [34]. In addition to the early identification of the case, triage allows notifying the medical staff and improves the timeliness of ED care and management to reduce the risks associated with the disease [36]. Our study showed a non-statistically significant downward trend in post-intervention mortality. This may be explained by the post intervention observation time of our study, possibly short to evaluate this outcome.

There is variable evidence regarding mortality in the population with ischemic CVA [1], [37], [38]. In a metaanalysis, Emberson found that performing reperfusion therapy increases the risk of fatal parenchymal hemorrhage in the first seven days of treatment [39]. In another study, Fonarow et al. evidenced an $11 \%$ decrease in in-hospital mortality due to any cause (OR $0.8995 \% \mathrm{Cl} 0.83-0.94)$, after implementing goodpractice measures, including triage protocols, to improve the quality of care in the management of CVA [40].

\section{Increase in the Reperfusion Therapy:}

Reperfusion therapy has modified the evolution of ischemic CVA, reducing the risk of death, disability and other adverse outcomes [31], [32]. One of the great challenges is the detection of the probable case during triage, to accelerate attention, define reperfusion therapy and reduce DTN [34]. Douglas et al. evidenced an increase in the frequency of use of rTPA in ischemic CVA when institutional protocols for care including triage protocols - existed [41]. Fonarow et al. also evidenced that, by implementing an institutional good-practice program in the management of CVA - including triage protocols - the proportion of patients receiving rTPA with DTN of less than 60 minutes increases, and the improvement of the state of independence was presented at hospital discharge, and decreased in-hospital mortality due to any cause [40]. Lindsberg et al. also evidenced a $20 \%$ increase in the use of rTPA in the population with CVA after having updated the triage protocol in an ED [35].

In our study, we evidence improvement in the timeliness of ED care, and an increase in the use of rTPA in the population evaluated, after the implementation of the triage policy, a statistically significant finding. Although the goal of implementing reperfusion therapy in the population with CVA is still far away, the impact of triage is evident. 


\section{STRENGTHS AND LIMITATIONS:}

To carry out the study, we defined a methodology that minimizes the risk of confusion bias due to variables related to the individual [10], [15], and a quality control was established to reduce confusion due to unmeasured variables [11].

The implementation of the TP proposed by the Colombian Ministry of Health was the only intervention of a regulatory order identified in the periods of time studied, related to the care of patients who consulted the EDs. However, during the time period of the study, the criteria for performing reperfusion therapy with rTPA were extended in Colombia, which may have predisposed to more timely care. And although the determination to receive the treatment does not necessarily depend on the TP, the use of rTPA can be considered as a co-intervention, since it is related to the outcome and its use was increased at the same time as the policy was implemented. This effect was controlled with a quality control group, in which there were no changes in these outcomes.

The intervention (the TP) did not affect the manner in which information was collected and stored in the institution. The data were collected electronically and automatically by the Information Management Office.

The main outcome (opportunity in care) was measured objectively, since it is a quantitative variable, and this was extracted directly from the hospital database. However, this information may be inaccurate in some circumstances, given that the recording of systematized attention times may be an unreliable representation of the actual care. And the size of the effect for the intervention was pre-specified, according to the simulation proposed by Zhang [21] in the determination of the sample size.

The limitations presented were the source of information was secondary, since it was recorded automatically in the electronic clinical history, from the completion of the triage to the beginning of the medical care, which can lead to an under-registration of this information; and second, the data in the control were only available as of January 2013.

\section{IMPLICATIONS FOR PRACTICE:}

The timeliness of ED care is critical in time-sensitive diseases, given that it can minimize the risk of adverse outcomes. Triage is the tool to determine this timeliness of ED care. Although the implementation of the structured five-level triage proposed by the Colombian Ministry of Health allowed improving timeliness of care in the ED for patients with ischemic CVA classified as Triage I and II, compared to Triage III, IV and V Categories. Strategies for quantification and improvement of the timeliness of ED care must be implemented.

\section{IMPLICATIONS FOR OTHER RESEARCH}

Although one of the critical times in the chain of care of the patient with CVA (from triage to medical care) was evaluated, it is necessary to quantify in greater depth the times of other moments in this same 
population (Door-CT Time; Door-to-Needle Time), which will permit establishing the quality of care for this population with greater precision. Other research derived from this work includes performing an ITS analysis with the CVA mortality data from across the country. This would increase the number of observations in each month by decreasing the variability of the estimate at each time point. Regarding the evaluation of the outcomes, in a future study it would be important to evaluate disability after the implementation of the triage policy.

\section{Declarations}

\section{Ethics approval and consent to participate}

This study was approved by the Technical and Ethical Committees of the hospitals where the study was conducted, IPS Universitaria Clínica León XIII and Hospital Pablo Tobón Uribe.

\section{Consent for publication}

Not aplicable

\section{Availability of data and materials}

The datasets generated during and/or analysed during the current study are available from the corresponding author on reasonable request.

\section{Competing interests}

The authors declares that they have no competing interests.

\section{Funding}

This study was funded by the University of Antioquia Faculty of Medicine

\section{Authors' contributions}

CEVB contributed with conception and design of the work:; the acquisition, analysis, and interpretation of data; and substantively revised the final paper.

DCAA contributed with analysis, and interpretation of data; and substantively revised the final paper.

JPA contributed with the acquisition of data; and substantively revised the final paper.

DFPL contributed with conception and design of the work; the analysis, and interpretation of data; and substantively revised the final paper.

All the authors have approved the submitted versión, and agreed to be personally accountable for the author's own contributions and to ensure that questions related to the accuracy or integrity of any part of 
the work, even ones in which the author was not personally involved, are appropriately investigated, resolved, and the resolution documented in the literature.

\section{Acknowledgments}

Our thanks to the IPS Universitaria - Clínica León XIII and Hospital Pablo Tobón Uribe (HPTU) in Medellín, Colombia, for providing the data for the study.

\section{References}

1. Mozaffarian D, et al., "Heart Disease and Stroke Statistics 2016 Update: A Report From the American Heart Association," Circulation p published online ahead of print December 16, 2015, doi:10.1161/CIR.0000000000000350.

2. Instituto Nacional de Salud de Colombia. "Carga de Enfermedades Crónicas No Transmisibles y Discapacidad en Colombia," Inf. Técnico Obs. Nac. Salud, vol. V Edición, 2015.

3. Feigin VL, Lawes CM, Bennett DA, Barker-Collo SL, Parag V. Worldwide stroke incidence and early case fatality reported in 56 population-based studies: a systematic review. Lancet Neurol. 2009;8(4):355-69. doi:10.1016/S1474-4422(09)70025-0.

4. O. N. de S. Instituto Nacional de Salud, "Quinto Informe ONS: carga de enfermedad por enfermedades crónicas no transmisibles y discapacidad en Colombia," Bogotá, 2015.

5. Stang AS, Crotts J, Johnson DW, Hartling L, Guttmann A. "Crowding Measures Associated With the Quality of Emergency Department Care: A Systematic Review," Acad Emerg Med, 643-56, 2015, doi:10.1111/acem.12682.

6. Mw K, Kd K, E F. Acute ischemic stroke - from symptom recognition to thrombolysis. Acta Neurol Scand. 2013;127(3):57-64. doi:10.1111/ane.12051.

7. Fonarow GC, et al. Improving Door-to-Needle Times in Acute Ischemic Stroke The Design and Rationale for the American Heart Association / American Stroke Association 's Target: Stroke Initiative. Stroke. 2011;42:2983-9. doi:10.1161/STROKEAHA.111.621342.

8. Ministerio de salud y proteccion social, Resolución 5596 del 2015, vol. 2015. Bogotá, Colombia, 2015, p. 5.

9. Montgomery DC. Design and Analysis of Experiments. Eighth Edi: John Wiley \& Sons, Inc; 2013.

10. Bernal JL, Cummins S, Gasparrini A. Interrupted time series regression for the evaluation of public health interventions: a tutorial. Int J Epidemiol. 2017;46(1):348-55. doi:10.1093/ije/dyw098.

11. Ramsay CR, Matowe L, Grilli R, Grimshaw JM, Thomas RE. "Interrupted time series designs in health technology assessment: lessons from two systematic reviews of behavior change strategies. " Int J Technol Assess Health Care. 2003;19(4):613-23. doi:10.1017/S0266462303000576. doi.

12. Wagner AK, Soumerai SB, Zhang F, Ross-Degnan D. Segmented regression analysis of interrupted time series studies in medication use research. J Clin Pharm Ther. 2002;27(4):299-309., ",, , , doi: 430 [pii]. 
13. Gillings D, Makuc D, Siegel E. Analysis of interrupted time series mortality trends: An example to evaluate regionalized perinatal care. Am J Public Health. 1981;71(1):38-46. doi:10.2105/AJPH.71.1.38.

14. $10.1093 / \mathrm{ije} /$ dyy 135

Bernal JL, Cummins S, Gasparrini A, "The use of controls in interrupted time series studies of public health interventions," Int. J. Epidemiol., no. July, pp. 2082-2093, 2018, doi: 10.1093/ije/dyy135.

15. Kontopantelis E, Doran T, Springate DA, Buchan I, Reeves D. Regression based quasi-experimental approach when randomisation is not an option: interrupted time series analysis. BMJ. 2015;350:2750. doi:10.1136/bmj.h2750.

16. Hopewell S, Ravaud P, Baron G. Effect of editors ' implementation of CONSORT guidelines on the reporting of abstracts in high impact medical journals: interrupted time series analysis. BMJ. 2012;344(4178):1-7. doi:10.1136/bmj.e4178.

17. Jandoc R, Burden AM, Mamdani M, Linda EL, Cadarette SM. Interrupted time series analysis in drug utilization research is increasing: systematic review and recommendations. J Clin Epidemiol. 2015;68:950-6. doi:10.1016/j.jclinepi.2014.12.018.

18. Morgan OW, Griffiths C, Majeed A. Interrupted Time-Series Analysis of Regulations to Reduce Paracetamol (Acetaminophen) Poisoning. Plos Med. 2007;4(4):105. doi:10.1371/journal.pmed.0040105.

19. Penfold RB, Zhang F. Use of Interrupted Time Series Analysis in Evaluating Health Care Quality Improvements. Acad Pediatr. 2013;13(6):S38-44. doi:10.1016/j.acap.2013.08.002.

20. Adesi BF, Gasparrini A, Vizzini L, Merletti F, Richiardi L. Decline in Rates of Hospital Admissions for Acute Coronary Events Following the Introduction of the Italian Smoking Regulation: A Country-Wide Study. PLoS One. 2011;6(3):129. doi:10.1371/journal.pone.0017419.

21. Zhang F, Wagner AK, Ross-degnan D. Simulation-based power calculation for designing interrupted time series analyses of health policy interventions. J Clin Epidemiol. 2011;64(11):1252-61. doi:10.1016/j.jclinepi.2011.02.007.

22. Cruz $\mathrm{M}$, Bender $\mathrm{M}, \mathrm{Ombao} \mathrm{H}$. A robust interrupted time series model for analyzing complex health care intervention data. Stat Med. 2017;36(29):4660-76. doi:10.1002/sim.7443.

23. Bender M, Ombao H, "A Robust Interrupted Time Series Model for Analyzing Complex Healthcare Intervention Data," ArXiv e-prints, pp. 1-29, 2017.

24. $10.1111 / 1742-6723.12426$

Ro YS, Do Shin S, Song KJ, Cha WC, Cho JS, "Triage-based resource allocation and clinical treatment protocol on outcome and length of stay in the emergency department," Emerg. Med. Australas., vol. 27, no. May, pp. 328-335, 2015, doi: 10.1111/1742-6723.12426.

25. Robinson DJ. An Integrative Review: Triage Protocols and the Effect on ED Length of Stay. J Emerg Nurs. 2013;39(4):398-408. doi:10.1016/j.jen.2011.12.016.

26. Farrohknia N, et al. "Emergency department triage scales and their components: a systematic review of the scientific evidence. " Scand J Trauma Resusc Emerg Med. 2011;19(1):42. doi:10.1186/1757- 
7241-19-42.

27. Article R. Modern triage in the emergency department. Dtsch Arztebl Int. 2010;107(50):892. doi:10.3238/arztebl.2010.0892.

28. Oredsson S, Jonsson H, Rognes J, Lind L, Göransson KE, Ehrenberg A. A systematic review of triagerelated interventions to improve patient flow in emergency departments. Scand J Trauma Resusc Emerg Med. 2011;19:43. doi:10.1186/1757-7241-19-43.

29. Murray M, Bullard M, Grafstein E. Revisions to the Canadian Emergency Department Triage and Acuity Scale Implementation Guidelines. Can J Emerg Med. 2004;6(6):421-7.

30. Bernstein SL, et al. The Effect of Emergency Department Crowding on Clinically Oriented Outcomes. Acad Emerg Med. 2009;16:1-10. doi:10.1111/j.1553-2712.2008.00295.x.

31. Saver JL. Time is brain - Quantified. Stroke. 2006;37(1):263-6. doi:10.1161/01.STR.0000196957.55928.ab.

32. Meretoja A, et al. Stroke thrombolysis: Save a minute, save a day. Stroke. 2014;45(4):1053-8. doi:10.1161/STROKEAHA.113.002910.

33. Xian Y, et al. Strategies used by hospitals to improve speed of tissue-type plasminogen activator treatment in acute ischemic stroke. Stroke. 2014;45(5):1387-95. doi:10.1161/STROKEAHA.113.003898.

34. Xian Y, et al., "Use of Strategies to Improve Door-to-Needle Times with Tissue-Type Plasminogen Activator in Acute Ischemic Stroke in Clinical Practice: Findings from Target: Stroke," Circ Cardiovasc Qual Outcomes, 10, 1, 2017, doi:10.1161/CIRCOUTCOMES.116.003227.

35. O. PJL H. M. K. L. V. M. K. and Kaste M. Door to thrombolysis: ER reorganization and reduced delays to acute stroke treatment. Neurology. 2006;67:334.

36. Hankey GJ, “Stroke. ” Lancet. 2016;389(10069):641-54. doi:10.1016/S0140-6736(16)30962-X.

37. Sauser K. Hospital Variation in Thrombolysis Times Among Patients With Acute Ischemic Stroke The Contributions of Door-to-Imaging Time and Imaging-to-Needle Time. JAMA Neurol. 2014;71(9):1155-61. doi:10.1001/jamaneurol.2014.1528.

38. Lavados PM, et al. Stroke epidemiology, prevention, and management strategies at a regional level: Latin America and the Caribbean. " Lancet Neurol. 2007;6:no. April, pp. 362-72. doi:10.1016/S14744422(07)70003-0.

39. Emberson J, et al. Effect of treatment delay, age, and stroke severity on the effects of intravenous thrombolysis with alteplase for acute ischaemic stroke: a meta-analysis of individual patient data from randomised trials. Lancet. 2014;384:1929-35. doi:10.1016/S0140-6736(14)60584-5.

40. Fonarow GC, et al. Door-to-Needle Times for Tissue Plasminogen Activator Administration and Clinical Outcomes in Acute Ischemic Stroke Before and After a Quality Improvement Initiative. JAMA. 2015;311(16):1632-40. doi:10.1001/jama.2014.3203.

41. Douglas VC, et al. Do the Brain Attack Coalition's criteria for stroke centers improve care for ischemic stroke? Neurology. 2005;64(3):422-7. doi:10.1212/01.WNL.0000150903.38639.E1. 
Figures

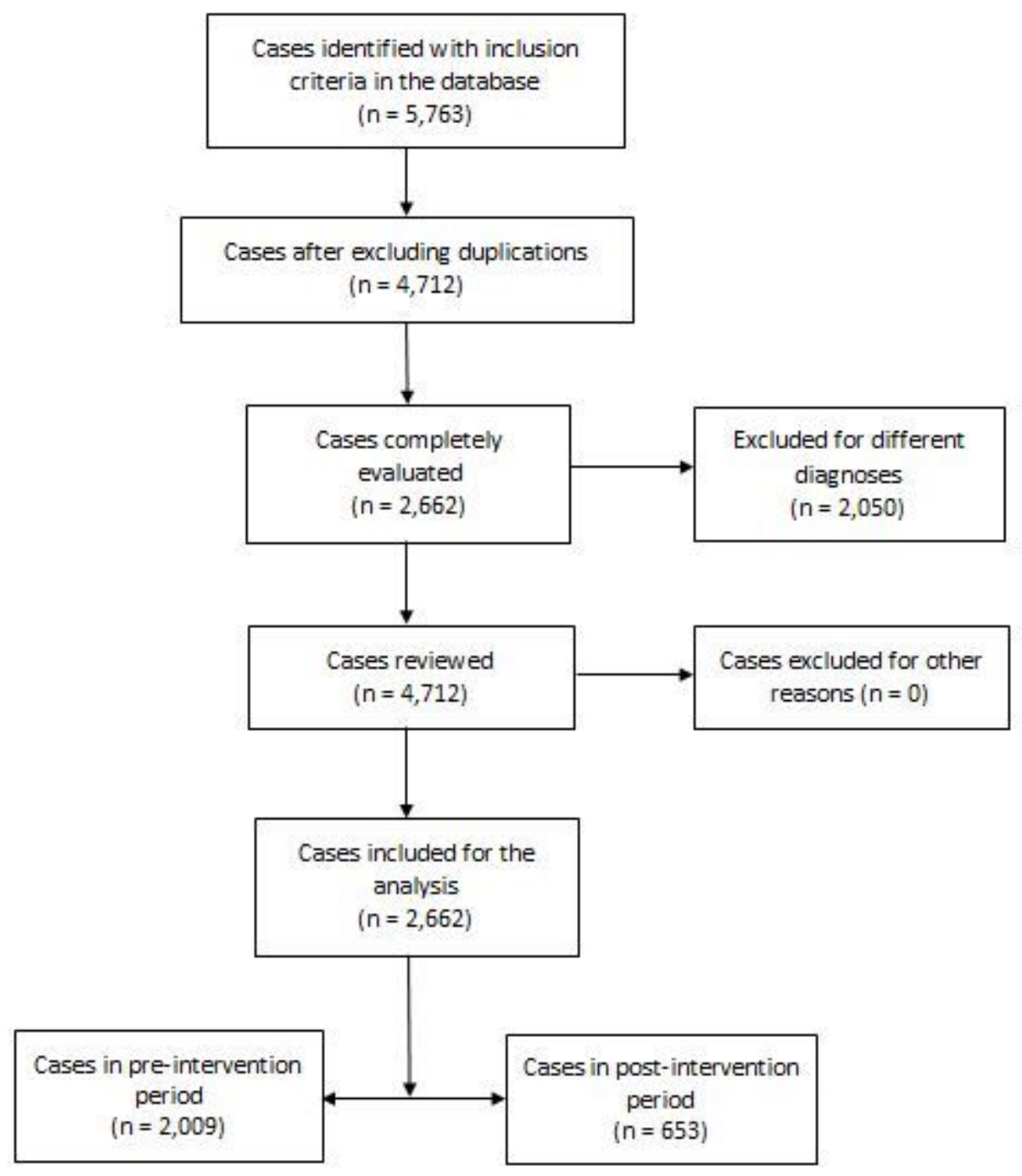

Figure 1

Diagram of recruitment of patients included and excluded in the time series 
Timeliness of care in ED for patients with ischemic CVA from 2011 to 2017

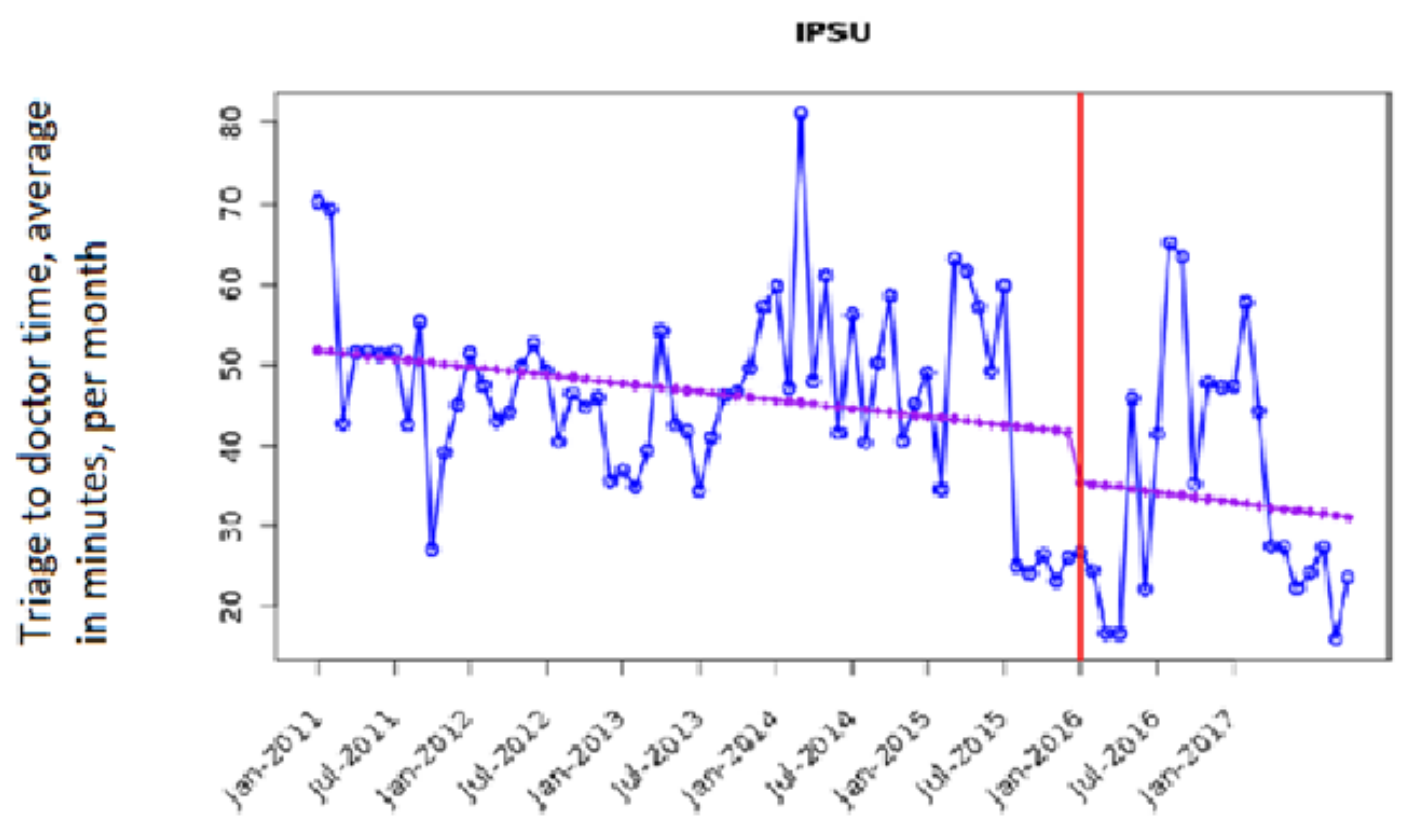

Figure 2

Timeliness of care in ED in minutes for patients with ischemic CVA by month

Timeliness of care in ED for patients with ischemic CVA in control group

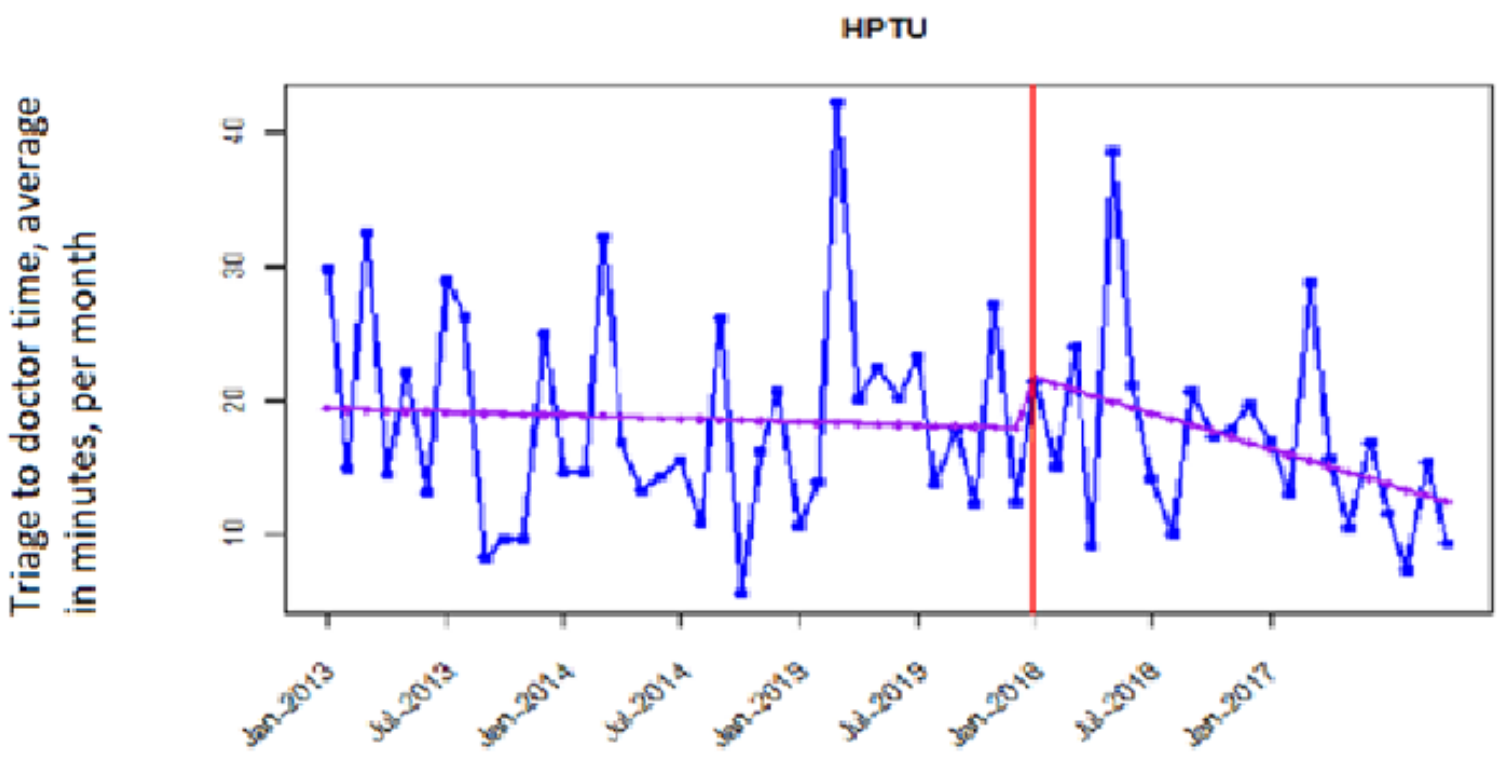


Figure 3

Timeliness of care in ED for patients with ischemic CVA, in control group

Timeliness fo care in ED for ischemic CVA triage I-II subgroup patients

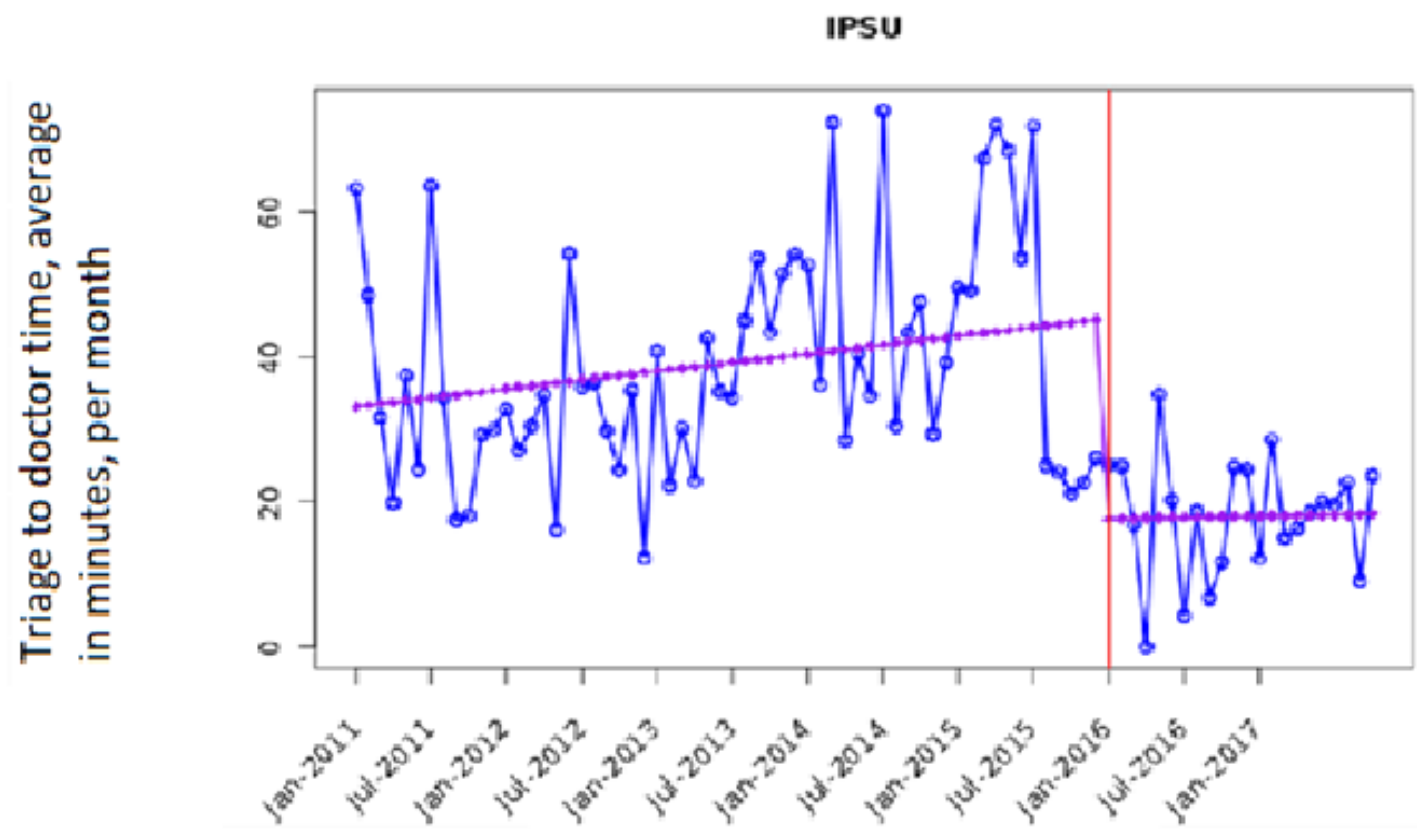

Figure 4

Timeliness of care in ED in intervention group for patients of the Triage I - II subgroup

\section{Supplementary Files}

This is a list of supplementary files associated with this preprint. Click to download.

- Appendix2.docx

- Appendix1.docx 